\title{
Disruption of gastrointestinal integrity in patients with HIV infection
}

Primary infection with HIV leads to severe depletion of CD4 T cells. This effect is conveniently measured in the blood compartment, but is even more pronounced in the gastrointestinal tract, which may be the initial site of acquisition of HIV for some people(1). Persistent HIV gastrointestinal infection impairs local immune defences, leads to immune activation and disrupts the epithelial barrier. The epithelial dysfunction is not completely repaired by antiretroviral therapy (ART) that is effective enough to suppress HIV viral load in the blood to undetectable levels(1). At the microscopic level, disruption of tight junctions between adjacent columnar epithelial cells is seen as a component. The resulting loss of integrity of the epithelium allows translocation of bacteria into the bloodstream and this has been investigated as a potential contributor to the chronic systemic inflammation seen in HIV patients(2).

These studies have now been supplemented by investigations to detect a viral pathogen in the gastrointestinal tract. Cytomegalovirus is found in the vast majority of HIV positive patients. Opportunistic disease, usually retinitis, caused by high viral loads of CMV are nowadays mostly prevented by ART, where this is available. However, there is extensive evidence that CMV infection, as opposed to overt disease, is not completely controlled by ART. It is known to infect endothelial, stromal and intestinal epithelial cells and may form ulcers to compromise the barrier function of the mucosa. It is known to disrupt tight junctions of retinal pigment epithelial cells, so might be able to do the same in epithelial cells of the gastrointestinal tract(3). It is possible therefore that it may contribute to the chronic gastrointestinal and systemic inflammation seen in HIV-positive patients.

A recent publication combined immunohistochemistry with in situ hybridisation on primary intestinal cells differentiated to form monolayers of polarised cells(4). In this system, CMV decreased trans-epithelial electrical resistance, a method used to assess the integrity of an epithelial monolayer. Permeability was also changed when assessed by the ability of dextran labelled with a fluorescent dye to migrate across cells held in the compartments of a transwell apparatus. Only a few cells were CMV positive, raising the question of how an infection of limited scale could produce an effect across the whole epithelium. A contribution from interleukin- 6 was identified, with the effect on epithelial integrity partially reduced by an antibody that blocked interleukin-6(4, 5). The antiviral drugs ganciclovir or letermovir reduced the effect of CMV on the epithelium. Parallel studies on biopsies of rectosigmoid tissue provided evidence that similar effects take place in vivo(4). CMV proteins were readily detected in untreated HIV positive, CMV seropositive individuals. The quantity of cells expressing CMV proteins was reduced, but not abolished, in patients receiving ART. Tight junctions were disrupted and CMV DNA was present, thereby confirming productive CMV infection. Rectosigmoid biopsies from HIV negative, CMV seropositive individuals did not contain CMV proteins in intestinal epithelial cells, although neighbouring cells stained positive, illustrating that the presence of HIV could alter the distribution of CMV. Finally, biopsies from an HIV positive case of CMV colitis showed damage to tight 
junctions which was no longer apparent in biopsies taken after a course of valganciclovir had been completed(4).

Overall, this effect on gastrointestinal permeability should be classified along with the "indirect effects" of CMV which contrast with its "direct effects" of causing end-organ diseases such as retinitis. The indirect effects were originally described in allograft recipients and referred to CMV triggering graft rejections, contributing to the chronic inflammation that causes accelerated atherosclerosis or diverting immune responses to facilitate secondary bacterial or fungal infections(6). The concept of indirect effects was subsequently discussed in HIV-positive patients as a way of explaining complex phenomena, including accelerated progression to full-blown AIDS and an excess of deaths, where it was called a cofactor effect $(7,8)$. Cohort studies report a significant association between CMV and mortality with deaths reduced in those allocated to receive systemic ganciclovir rather than local therapy for retinitis(9-11). A randomised placebo-controlled trial in HIV positive individuals showed that valganciclovir decreased the abundance of the activated $T$ cells that are the hallmark of chronic inflammation(12).

It is clear from this new paper and others that there are multiple ways for this opportunistic virus to interact with different organ systems to produce disease without declaring its presence. This research is to be welcomed as a way of starting to tease out the contributions that this common virus makes to medically important conditions in patients with complex medical backgrounds. The results demonstrate clearly that CMV persistence in the gastrointestinal tract can, like bacterial infections, provoke chronic, systemic inflammation in HIV positive individuals. The challenge now is to determine if CMV represents just one of many examples of an infectious agent provoking chronic inflammation or whether CMV induced cytokine response is a central feature of pathogenesis that allows bacterial antigens to cross into the systemic circulation. In other words, would reduction of CMV gastrointestinal infection in a randomised placebo-controlled trial of an anti-CMV drug like valganciclovir in HIV positive individuals maintain normal intestinal barrier function? If so, would this reduce the adverse effects attributed both to CMV and to those caused by bacteria?

PD Griffiths

$\mathrm{ART}=$ anti-retroviral therapy

1. Brenchley JM, Schacker TW, Ruff LE, Price DA, Taylor JH, Beilman GJ, et al. CD4+ T cell depletion during all stages of HIV disease occurs predominantly in the gastrointestinal tract. The Journal of experimental medicine. 2004 Sep 20;200(6):749-59. PubMed PMID: 15365096. Pubmed Central PMCID: 2211962.

2. Jiang W, Lederman MM, Hunt P, Sieg SF, Haley K, Rodriguez B, et al. Plasma levels of bacterial DNA correlate with immune activation and the magnitude of immune restoration in persons with antiretroviral-treated HIV 
infection. The Journal of infectious diseases. 2009 Apr 15;199(8):1177-85.

PubMed PMID: 19265479. Pubmed Central PMCID: 2728622.

3. Tugizov S, Maidji E, Pereira L. Role of apical and basolateral

membranes in replication of human cytomegalovirus in polarized retinal pigment epithelial cells. The Journal of general virology. 1996 Jan;77 ( Pt 1):61-74. PubMed PMID: 8558129.

4. Maidji E, Somsouk M, Rivera JM, Hunt PW, Stoddart CA. Replication of CMV in the gut of HIV-infected individuals and epithelial barrier dysfunction. PLoS pathogens. 2017 Feb;13(2):e1006202. PubMed PMID: 28241080. Pubmed Central PMCID: 5328284.

5. $\quad$ Dupont L, Reeves MB. Cytomegalovirus latency and reactivation: recent insights into an age old problem. Reviews in medical virology. 2016 Mar;26(2):75-89. PubMed PMID: 26572645.

6. Rubin $\mathrm{RH}$. The indirect effects of cytomegalovirus infection on the outcome of organ transplantation. JAMA. 1989;261(24):3607-9.

7. Webster A, Grundy JE, Lee CA, Emery VC, Cook DG, Kernoff PB, et al. Cytomegalovirus infection and progression to AIDS. Lancet. 1989;2(8664):681.

8. Griffiths PD. Studies of viral co-factors for human immunodeficiency virus in vitro and in vivo. JGenVirol. 1998;79 ( Pt 2):213-20.

9. Deayton JR, Sabin CA, Johnson MA, Emery VC, Wilson P, Griffiths PD. Importance of cytomegalovirus viraemia in risk of disease progression and death in HIV-infected patients receiving highly active antiretroviral therapy. Lancet. 2004;363(9427):2116-21.

10. Spector SA, Wong R, Hsia K, Pilcher M, Stempien MJ. Plasma cytomegalovirus (CMV) DNA load predicts CMV disease and survival in AIDS patients. JClinInvest. 1998;101(2):497-502.

11. Kempen JH, Jabs DA, Wilson LA, Dunn JP, West SK, Tonascia J. Mortality risk for patients with cytomegalovirus retinitis and acquired immune deficiency syndrome. ClinInfectDis. 2003;37(10):1365-73.

12. Hunt PW, Martin JN, Sinclair E, Epling L, Teague J, Jacobson MA, et al. Valganciclovir reduces T cell activation in HIV-infected individuals with incomplete CD4+ T cell recovery on antiretroviral therapy. JlnfectDis. 2011;203(10):1474-83. 